\title{
LL-37 secreted by epithelium promotes fibroblast collagen production: a potential mechanism of small airway remodeling in chronic obstructive pulmonary disease
}

\author{
Congcong Sun ${ }^{1}$, Maoxiang Zhu², Zhihua Yang ${ }^{2}$, Xiujie Pan ${ }^{2}$, Yuke Zhang ${ }^{1}$, Qin Wang ${ }^{1}$ and Wei Xiao ${ }^{1}$
}

Emerging evidence suggests that the process of small airway remodeling is mediated by profibrotic growth factors produced by epithelium, which are capable of activating the underlying mesenchymal cells with excessive collagen production. It has been demonstrated that human cathelicidin antimicrobial protein LL-37 is highly expressed in small airway epithelium from COPD patients. However, it is unknown whether the increased levels of LL-37 in epithelium are involved in the pathogenesis of small airway remodeling in COPD. In this study, we examined the expression of LL-37 in small airways from smokers with COPD and controls (non-smokers and smokers without COPD) by immunohistochemistry, and then the association between LL-37 expression in epithelium and the structural changes of small airway remodeling was analyzed. In vitro, the effect of CSE-induced epithelial secretion of LL-37 on collagen production in human lung fibroblasts (HFL-1 cell line) was studied in a co-culture system. Finally, the signaling pathways involved in the effect of LL-37 on fibroblast collagen production were evaluated. The results showed that LL-37 immunoreactivity in airway epithelium was significantly elevated in smokers with COPD compared with controls. In addition, the magnitude of LL-37 expression in epithelium was positively correlated with airway wall thickness and collagen deposition. In vitro, CSE-induced epithelial secretion of LL-37 promoted fibroblast collagen production. Finally, we showed that formyl peptide receptor-like 1 (FPRL1)-dependent extracellular signal-regulated kinase (ERK) signaling pathway was essential for LL-37-induced collagen production in HFL-1 cells. These results suggest that after cigarette smoke exposure, the increased levels of LL-37 in airway epithelium could stimulate collagen production in the underlying lung fibroblasts and may contribute to small airway remodeling in COPD.

Laboratory Investigation (2014) 94, 991-1002; doi:10.1038/labinvest.2014.86; published online 23 June 2014

Chronic obstructive pulmonary disease (COPD) is a major cause of chronic morbidity and mortality worldwide, which is predicted to become the third leading cause of death globally by $2020 .{ }^{1}$ Small airway remodeling is a prominent feature of COPD, which is characterized by thickening of the airway wall with increased collagen deposition. In the 1960s, Hogg first introduced the concept of small airway remodeling and demonstrated that small airways (with internal diameter of $\leq 2 \mathrm{~mm}$ ) were the major site of resistance in COPD. ${ }^{2}$ Since then, there has been a growing interest in the study of small airways. However, due to lack of in vivo sampling and a practical tool for evaluation of their function, ${ }^{3}$ the molecular mechanism of small airway remodeling is unclear. Recent studies propose that small airway remodeling is mediated through profibrotic mediators (cytokines and growth factors) produced by epithelium, which are capable of activating subepithelial fibroblasts with increased collagen deposition. ${ }^{4-6}$

A number of studies have suggested that antimicrobial peptides (AMPs) are associated with the development of COPD. ${ }^{7,8}$ AMPs, including the defensin and cathelicidin family, ${ }^{9}$ are small peptides that represent essential elements of innate immunity. LL-37 is the only member of human cathelicidin family, which is shown to be widely expressed in neutrophils ${ }^{10}$ and epithelial cells of the conducting airways, the urinary tract, and the gastrointestinal tract. ${ }^{11-13}$ In addition to antimicrobial activities, LL-37 has pleiotropic

\footnotetext{
'Department of Respiratory Medicine, Qilu Hospital, Shandong University, Jinan, China and ${ }^{2}$ Department of Radiation Toxicology and Oncology, Beijing Institute of Radiation Medicine, Beijing, China

Correspondence: Professor W Xiao, Department of Respiratory Medicine, Qilu Hospital, Shangdong University, 107 Wenhua West Road, Jinan 250012, China.

E-mail: xiaowei4226@163.com

Received 12 April 2014; revised 27 May 2014; accepted 30 May 2014
} 
activities, including chemotaxis, ${ }^{14}$ angiogenesis, ${ }^{15}$ and wound repair, ${ }^{16}$ indicating the involvement of LL-37 in inflammatory and tissue remodeling processes. ${ }^{7}$ It has been reported that the expression of LL-37 is significantly elevated in induced sputum and bronchoalveolar lavage fluid from COPD patients. ${ }^{17-19}$ In addition, our previous study observed a significant increase of LL-37 in airway epithelium from COPD patients and demonstrated that LL-37 was implicated in the pathogenesis of airway inflammation and airway mucus overproduction..$^{20,21}$ However, there is no evidence that LL-37 secreted by epithelium is involved in small airway remodeling in COPD. Recently, LL-37 has been reported to show a tendency for increased expression of the alpha 1(I) collagen gene (COL1A1) in human dermal fibroblasts at higher concentrations, ${ }^{22}$ whereas the effect of LL-37 on collagen production in human lung fibroblasts has not been explored. Considering the overprduction of LL-37 in airway epithelium and its effect on collagen production in dermal fibroblasts, it is reasonable to hypothesize that increased levels of LL-37 in epithelium are able to induce collagen production in adjacent lung fibroblasts and then contribute to small airway remodeling.

In this study, we assessed the association between LL-37 expression in epithelium and the structural changes of small airway remodeling in smokers with COPD and controls. In addition, the effect of CSE-induced epithelial secretion of LL37 on fibroblast collagen production was evaluated in a coculture system. Finally, the potential mechanism of LL-37 action on collagen production in human lung fibroblasts was explored. Our results show that epithelial production of LL-37 after cigarette smoke exposure promotes fibroblast collagen production, which could be involved in the pathogenesis of small airway remodeling in COPD.

\section{MATERIALS AND METHODS Subjects}

Three groups of subjects were recruited for this study, including 20 non-smokers without COPD, 22 smokers without COPD, and 18 smokers with COPD. All subjects were patients who underwent resection of pulmonary nodules, and samples were obtained at Qilu Hospital (Jinan, China). For each subject, two tissue blocks (sample size 15-25 mm) were taken from the subpleural parenchyma of the lobe obtained in surgery, at least $5 \mathrm{~cm}$ far from the margin of the diseased regions. The diagnosis of COPD was made according to the guidelines of the Global Initiative for Chronic Obstructive Lung Disease. ${ }^{23}$ Before surgery, the medical history was assessed, and pulmonary function was measured using a spirometer (MasterScreen Series; Jaeger, Hoechberg, Germany). COPD patients were defined as the subjects who has dyspnea, chronic cough, and/or sputum production and a history of exposure to risk factors for the disease in the presence of a postbronchodilator $\mathrm{FEV}_{1} / \mathrm{FVC}<0.70$ confirming the airflow limitation. Patients with comorbidities, including asthma, interstitial lung diseases, heart failure, and neuromuscular disease, were excluded. None of the subjects had suffered from any respiratory tract infection or received any glucocorticoids or antibiotics during the month preceding the study. The study was approved by the Ethics Committee of Shandong University, and all participants signed the informed consent.

\section{Histological and Morphological Analysis}

Specimens of formalin-fixed tissue were embedded in paraffin, sectioned $(4 \mu \mathrm{m})$, and stained with hematoxylin-eosin for morphometric measurement and sirius red for assessment of collagen. Histological analysis was carried out manually using the Image-Pro Plus 5.0 software (Media Cybernetics, Silver Spring, MD, USA) on the digital images of the small airways $(<2 \mathrm{~mm}$ in diameter $)$ in a blind manner. Four randomly chosen fields per slide at 400 -fold magnification were examined. The following parameters were assessed: the length of the basement membrane $(\mathrm{Pbm})$, the area defined by the basement membrane (Abm) and the area defined by the total adventitial perimeter (Ao). The airway wall thickness was calculated as the total bronchial wall area (WAt, WAt $=$ Ao-Abm) normalized to the squared $\mathrm{Pbm}$ as previously described. ${ }^{24}$ For the quantification of collagen deposition, the area of collagen around the small airway wall was normalized to $\mathrm{Pbm}$.

\section{Immunohistochemistry}

Immunostaining for LL-37 was performed as described previously. ${ }^{20}$ Tissue sections were deparaffined and stained with rabbit anti-LL-37 antibody (Abcam, Cambridge, MA, USA) at 1:300 dilution. After incubation to horseradish peroxidase (HRP)-conjugated anti-rabbit antibody (1:1000, ZhongShan Biotech, Beijing, China), color was developed using diaminobenzidine. In the negative controls, the primary antibody was substituted with phosphate-buffered saline (PBS). Immunochemistry staining of lung tissue sections was evaluated at 400 -fold magnification in four random fields per section by an investigator who was blinded to the data. The mean staining density of LL-37 in airway epithelium was analyzed using the Image-Pro Plus 5.0 software as described previously. ${ }^{25}$ Briefly, integrated optical density (IOD) and stained area $\left(\mu \mathrm{m}^{2}\right)$ for positively stained epithelium were measured manually by the Image-Pro Plus 5.0 software. Results were calculated as the average IOD per stained area $\left(\mu \mathrm{m}^{2}\right)$ (IOD/area) for positive staining.

\section{Cell Culture and Cell Viability}

Human bronchial epithelial cells 16HBE14o (16HBE) and human fetal lung fibroblasts (HFL-1) were obtained from the American Type Culture Collection (ATCC). All cell lines were cultured in Dulbecco's modified Eagle's medium (DMEM) containing $10 \%$ fetal bovine serum, 100 units $/ \mathrm{ml}$ penicillin, and $100 \mu \mathrm{g} / \mathrm{ml}$ streptomycin. Cells were incubated at $37^{\circ} \mathrm{C}$ in a humidified $5 \% \mathrm{CO}_{2}$ atmosphere. Cell viability was assessed 
by a 3-(4,5-dimethylthiazol-2-yl)-2,5-diphenyl tetrazolium bromide (MTT, Sigma, USA) assay as described previously. ${ }^{26}$

\section{Preparation of CSE}

CSE was prepared by a modification described previously. ${ }^{27}$ Briefly, five research-grade cigarettes (3R4F, University of Kentucky, USA) without filters were combusted with an experimental apparatus driven by a peristaltic pump, and the smoke was bubbled through $10 \mathrm{ml}$ of PBS. The suspension was adjusted to $\mathrm{pH} 7.2$ and filtered through a $0.22-\mu \mathrm{m}$ pore filter to remove bacteria. This solution was considered to be $100 \%$ CSE and prepared within $30 \mathrm{~min}$ of each experiment.

\section{Immunofluorescence}

16HBE cells were seeded on chamber slides in serum-free medium. As a subconfluent culture was reached, cells were incubated with different concentrations of CSE for $48 \mathrm{~h}$, washed in PBS, and fixed in 4\% paraformaldehyde for $20 \mathrm{~min}$. Cells were permeabilized with $1 \%$ Triton X100 for 20 min and further incubated with rabbit anti-LL-37 antibody (1:200) overnight at $4{ }^{\circ} \mathrm{C}$. After washing, cells were incubated with goat anti-rabbit IgG-FITC (1:400) for $1 \mathrm{~h}$. Nuclei were counterstained with 4,6-diamidino-2-phenylindole (Sigma, USA) for $5 \mathrm{~min}$ in the dark. Images were taken with a Zeiss laser scanning confocal microscope (Carl Zeiss, Jena, Germany) with the Zeiss LSM 510 software, version 4.2.

\section{Co-Culture of 16HBE/HFL-1 and CSE Exposure}

Co-culture experiments were performed in six-well transwell plates (Corning, USA). 16HBE cells were plated at a density of $5 \times 10^{4}$ cells $/ \mathrm{ml}$ on transwell inserts containing a $0.4-\mu \mathrm{m}$ plastic membrane filter and submerged in the culture medium. HFL- 1 cells $\left(1 \times 10^{5}\right.$ cells $\left./ \mathrm{ml}\right)$ were grown separately in the lower chamber of transwell plates. The cultured cells were maintained in DMEM in the absence of serum. On reaching near confluence, cells were exposed to CSE in the presence or absence of anti-LL-37 neutralizing antibody at $1 \mu \mathrm{g} / \mathrm{ml}$ for $48 \mathrm{~h}$. Anti-mouse IgG was used as an unspecific antibody in this study. Alternatively, HFL-1 cells were cultured in the lower chamber of six-well transwell plates without co-existence with $16 \mathrm{HBE}$ cells and were stimulated with CSE at the same concentration for $48 \mathrm{~h}$.

\section{Treatment of HFL-1 Cells}

HFL-1 cells were starved $(24 \mathrm{~h})$ in a serum-free DMEM medium in $25-\mathrm{cm}^{2}$ flasks. On reaching near confluence, cells were incubated with the indicated doses of synthetic LL-37 (sequence $5^{\prime}$-LLGDFFRKSKEKIGKEFKRIVQRIKDFLRNLVP RTES-3', GL Biochem, Shanghai, China), a scrambled version of LL-37 (sLL-37, sequence 5'-RSLEGTDRFPFVRLKNSRKL EFKDIKGIKREQFVKIL-3', GL Biochem, Shanghai, China), or transforming growth factor- $\beta 1$ (TGF- $\beta 1$, Sigma, USA). Alternatively, cells were pretreated with p38 inhibitor, SB203580 $(10 \mu \mathrm{M})$, JNK inhibitor, SP600125 $(20 \mu \mathrm{M})$, ERK inhibitor, PD98059 $(10 \mu \mathrm{M})$, or formyl peptide receptor-like 1
(FPRL1) antagonist WRW4 (sequence TATTTT, GL Biochem, Shanghai, China) $2 \mathrm{~h}$ before LL-37 stimulation.

\section{Measurement of Total Collagen}

Total soluble collagen in the supernatant was quantified by the Sircol soluble collagen assay (Biocolor Ltd., Newtownabbey, UK) as described previously. ${ }^{28}$ One milliliter of Sircol dye was added to $100 \mu \mathrm{l}$ supernatant, followed by incubation under gentle rotation for $30 \mathrm{~min}$ at room temperature. After centrifugation for $10 \mathrm{~min}$ at $12000 \mathrm{rpm}$, the collagen-bound dye was redissolved with $250 \mu \mathrm{l}$ Sircol alkali reagent, and the absorbance was measured at $540 \mathrm{~nm}$.

RNA Isolation and Real-Time Reverse Transcription-PCR Reverse transcription (RT) and real-time PCR (PCR) was performed as described previously. ${ }^{29}$ After HFL-1 cells were treated with synthetic LL-37 for $48 \mathrm{~h}$, total RNA was isolated using the Trizol reagent and stored at $-20^{\circ} \mathrm{C}$ until use. One microgram of total RNA was reverse transcribed to cDNA by using a RNA-PCR kit (Promega, Madison, WI, USA) according to the manufacturer's recommendations. PCR amplifications were performed using $2 \mu \mathrm{l}$ of cDNA, $10 \mu \mathrm{l}$ of 2X GoTaq Green Master Mix, and $0.5 \mu \mathrm{M}$ of each primer in a $20-\mu$ l reaction volume. All the primers were synthesized by Sangon Biotech Co, Ltd. (Shanghai, China) and are listed in Table 1. RCR conditions were $95^{\circ} \mathrm{C} 10 \mathrm{~min}, 30$ cycles of $95^{\circ} \mathrm{C}$ $30 \mathrm{~s}, 55^{\circ} \mathrm{C} 20 \mathrm{~s}, 68^{\circ} \mathrm{C} 20 \mathrm{~s}$, and a final cycle of $72^{\circ} \mathrm{C}$ for $10 \mathrm{~min}$. The relative expression of each target mRNA was computed from the target threshold cycle $\left(\mathrm{C}_{\mathrm{T}}\right)$ values and the GAPDH $C_{T}$ values using the standard curve method.

\section{Western Blotting Assay}

Assessment of ERK phosphorylation following stimulation with LL-37 alone and in the presence of specific inhibitors was performed using western blotting. Total cell protein $(20 \mu \mathrm{g})$ was separated by $10 \%$ SDS-polyacrylamide gel electrophoresis, transferred to a PVDF membrane, and immunobloted with primary antibodies specific for ERK and phosphorylated ERK (pERK). Signals were detected following incubation with HRP-conjugated secondary antibody and chemiluminescence (Cell Signaling Technology, USA).

\section{Table 1 Primer sequences used for PCR amplification}

\begin{tabular}{llll}
\hline Gene & Strand & Sequence $5^{\prime} \rightarrow 3^{\prime}$ & Gene Bank \\
COL1A1 & F & GGGCAAGACAGTGATTGATA & JX573431 \\
& R & ACGTCGAAGCCGAATCCT & \\
COL3A1 & F & AGGTCCTGCGGGTAACACT & NM_000090 \\
& R & ACTTCACCCTTGACACCCTG & \\
GAPDH & F & GTGGAGTCCACTGGCGTCTT & J04038 \\
& R & GCAAATGAGCCCAGCCTTC &
\end{tabular}




\section{Statistical Analysis}

All data were expressed as mean \pm s.d. The nonparametric tests (Kruskal-Wallis and Mann-Whitney $U$ ) were used for comparisons between patient groups. $\chi^{2}$ test was used to compare categorical variables. The Spearman's test was used for correlation analysis between the two data. Comparisons of the in vitro data between multiple treatment groups were performed by one-way ANOVA. $P<0.05$ was considered statistically significant. Statistical analysis was carried out in the SPSS 16.0 software.

\section{RESULTS}

\section{Clinical Data}

Table 2 shows the main clinical and functional characteristics of the subjects in the study. All patients were well balanced by age and gender. Smoking histories were not significantly different between smokers and smokers with COPD. The forced expiratory volume in $1 \mathrm{~s}\left(\mathrm{FEV}_{1}\right)$ of predicted and the $\mathrm{FEV}_{1} /$ forced vital capacity (FVC) ratio were significantly lower in smokers with COPD compared with non-smokers and smokers without COPD $(P<0.01)$.

\section{Morphometric Analysis of Small Airway Remodeling}

The thickness of the airway wall from smokers with COPD was significantly elevated in comparison with non-smokers and smokers without COPD (Figure 1). Smokers with COPD also showed a tendency for an increased collagen deposition in small airways compared with non-smokers and smokers without COPD (Figure 2). No differences in the airway wall

\section{Table 2 Clinical and functional characteristics of the} population studied

\begin{tabular}{lccc}
\hline & Non-smokers & Smokers & $\begin{array}{c}\text { Smokers } \\
\text { with COPD }\end{array}$ \\
\hline Subjects ( $n$ ) & 20 & 22 & 18 \\
Age (years) & $54(11)$ & $57(10)$ & $59(9)$ \\
Sex (female/male) & $4 / 16$ & $1 / 21$ & $0 / 18$ \\
Smoking history (pack-years) & 0 & $25(13)$ & $39(27)$ \\
FEV 1 (\% predicted) & $95(14)$ & $99(10)$ & $69(17)^{\mathrm{a}, \mathrm{b}}$ \\
FEV 1 /FVC \% & $85(9)$ & $79(6)$ & $61(6)^{\mathrm{a}, \mathrm{b}}$ \\
& & & \\
GOLD stage & - & - & 0 \\
1 & - & - & 14 \\
2 & - & - & 4 \\
3 & - & - & 0 \\
\hline
\end{tabular}

Abbreviations: COPD, chronic obstructive pulmonary disease; FEV ${ }_{1}$, forced expiratory volume in one second; FVC, forced vital capacity.

Data are expressed as mean (s.d.).

${ }^{\mathrm{a}} P<0.01$ vs non-smokers.

${ }^{\mathrm{b}} P<0.01$ vs smokers without COPD. thickness and collagen deposition were found between nonsmokers and smokers without COPD $(P>0.05$ and $P>0.05$, respectively).

\section{Immunoreactivity of LL-37 in Small Airways}

The LL-37 immunostaining was localized in airway epithelial cells and subepithelial cells (inflammatory cells and fibroblasts). In non-smokers, a faint staining of LL-37 was seen in small airways (Figure 3a). There was a significant increase in LL-37 mean staining density in airway epithelium of smokers without COPD and smokers with COPD, especially in smokers with COPD (Figure 3b).

\section{Correlation Analysis}

To explore the possible role of epithelium-derived LL-37 in small airway remodeling, we assessed the relationships between LL-37 expression in epithelium and the structural changes of small airway remodeling. Among all the subjects, LL-37 immunoreactivity in epithelium was positively correlated with airway wall thickness $(r=0.62, \quad P<0.01$, Figure 4a). A similar correlation was also found between the epithelial expression of LL-37 and collagen deposition in small airways ( $r=0.73, P<0.01$, Figure $4 \mathrm{~b})$.

\section{CSE-Induced Epithelial Secretion of LL-37 Elevated Fibroblast Collagen Production in Co-Culture}

CSE has been extensively used to model cigarette smoke exposure in vitro. To investigate whether cigarette smoke induced LL-37 expression in bronchial epithelial cells, 16HBE cells were stimulated with CSE (2-6\%) for $48 \mathrm{~h}$, and LL-37 levels were examined by immunofluorescence. It was observed that the exposure to CSE resulted in a substantial enhancement of LL-37 staining in the cytoplasm of the 16HBE cells (Figure 5).

We next evaluated the effect of CSE-induced epithelial secretion on fibroblast collagen production in co-cultures of 16HBE and HFL-1 cell lines. After exposure to CSE for $48 \mathrm{~h}$, a significant increase of total collagen production was observed in a dose-dependent manner. To determine whether epithelial secretion of LL-37 was involved in fibroblast collagen production, an anti-LL-37 neutralizing antibody (preventing the effect of secreted LL-37 in the supernatant) was added to the co-cultured cells. As shown in Figure 6a, neutralization of LL-37 activity partially abolished the production of collagen. However, anti-mouse IgG had no effect on collagen production. To evaluate the direct effect of CSE on fibroblast collagen production, we exposed CSE to HFL-1 cells at the same concentration and found that CSE did not influence collagen production significantly (Figure 6b).

\section{LL-37 Increased Collagen Protein and Gene Expression in Human Lung Fibroblasts}

To study the effect of LL-37 on collagen expression, HFL-1 cells were incubated with synthetic LL-37 for $48 \mathrm{~h}$. As shown in Figure 7a, synthetic LL-37 induced a dose-dependent 

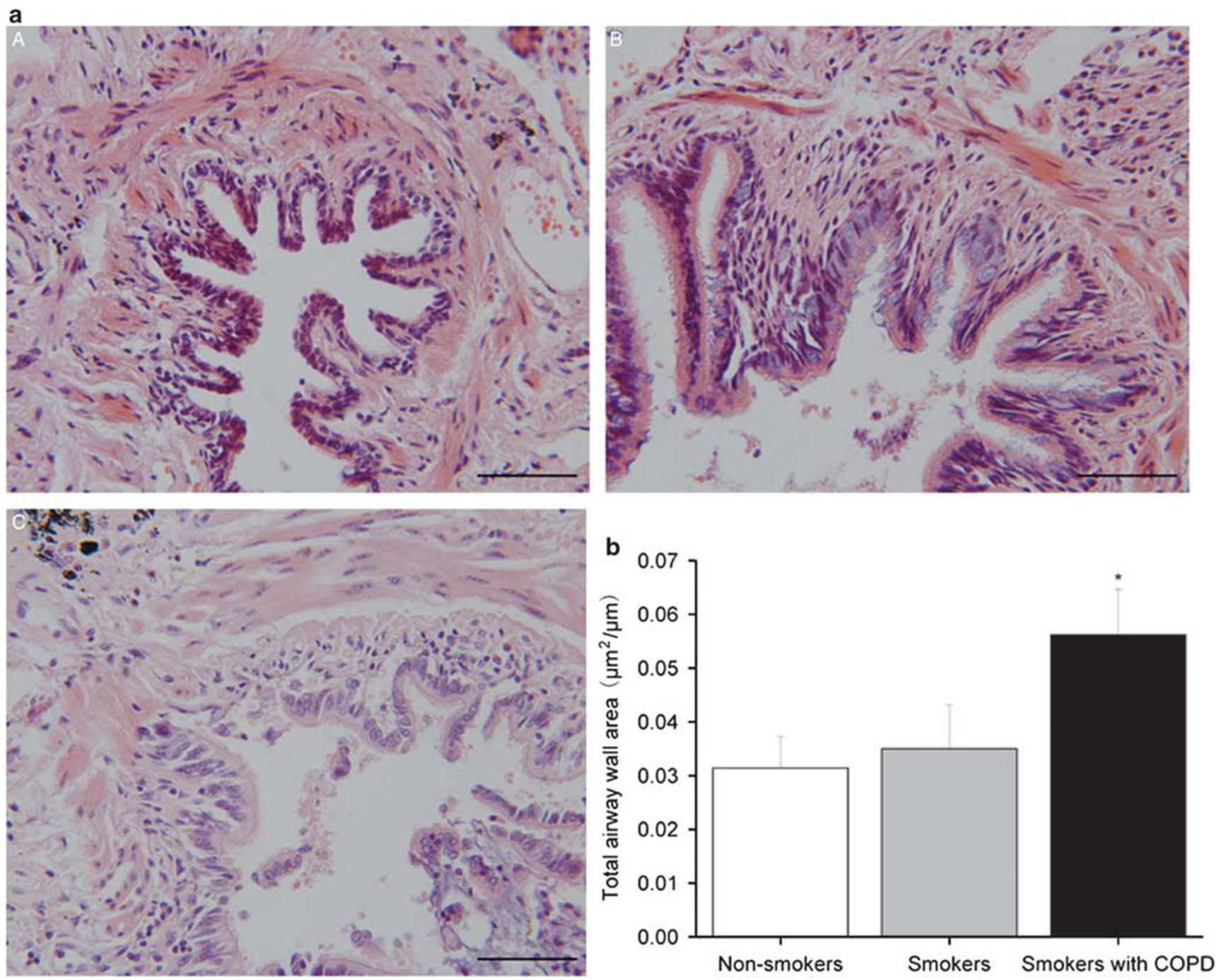

Figure 1 Airway wall thickness of non-smokers, smokers, and smokers with COPD. (a) Representative photomicrographs of hematoxylin-eosin staining in small airways from non-smokers (A), smokers (B), and smokers with COPD (C) (all images are $\times 400$ magnification, scale bars $=50 \mu \mathrm{m})$. (b) Airway wall thickness calculated as the total airway wall area (WAt) normalized to the squared length of the basement membrane (Pbm) in subjects of different groups. ${ }^{*} P<0.01$, compared with non-smokers.

increase in total collagen content. In contrast, sLL-37 had no significant effect on collagen synthesis, indicating that LL-37induced collagen production is a specific effect of the peptide. TGF- $\beta 1$ is an important profibrotic growth factor in tissue fibrosis by stimulating collagen synthesis in fibroblasts. As expected, TGF- $\beta 1$ resulted in a significant increase in total soluble collagen expression compared with the control group.

RT-PCR was carried out to evaluate the RNA transcriptional levels of COL1A1 and COL3A1, and mRNA levels were assessed using $\mathrm{C}_{\mathrm{T}}$ values. In our study, $\mathrm{C}_{\mathrm{T}}$ values for target and control genes are as follows: type I collagen gene (COL1A1) (median, 21; range, 20-22), type III collagen gene (COL3A1) (median, 22; range, 21-23), and GAPDH (median, 14; range, 13-15). Relative gene expression was calculated according to the $2^{-\Delta \Delta C}$ T method. As shown in Figure $7 \mathrm{~b}$, a significant upregulation of COL1A1 and
COL3A1 expression in HFL-1cells was observed after synthetic LL-37 stimulation.

MTT assay showed that exposure to low concentrations of synthetic LL-37 did not influence cell viability until exposure to concentration $>200 \mathrm{ng} / \mathrm{ml}$. Cells treated with $1000 \mathrm{ng} / \mathrm{ml}$ synthetic LL-37 for $48 \mathrm{~h}$ exhibited $8.31 \%$ decrease in cell viability compared with the control group (Figure 7c).

\section{FPRL-1 was Required for LL-37-Induced Collagen Expression in HFL-1 Cells}

As the effects of LL-37 have been demonstrated to be initiated by binding to specific receptors, we examined the involvement of FPRL1 in LL-37-induced collagen production in HLF-1 cells. Pretreatment of cells with WRW4, a specific inhibitor of the FPRL1, could suppress synthetic LL-37-induced collagen production in a concentration-dependent 

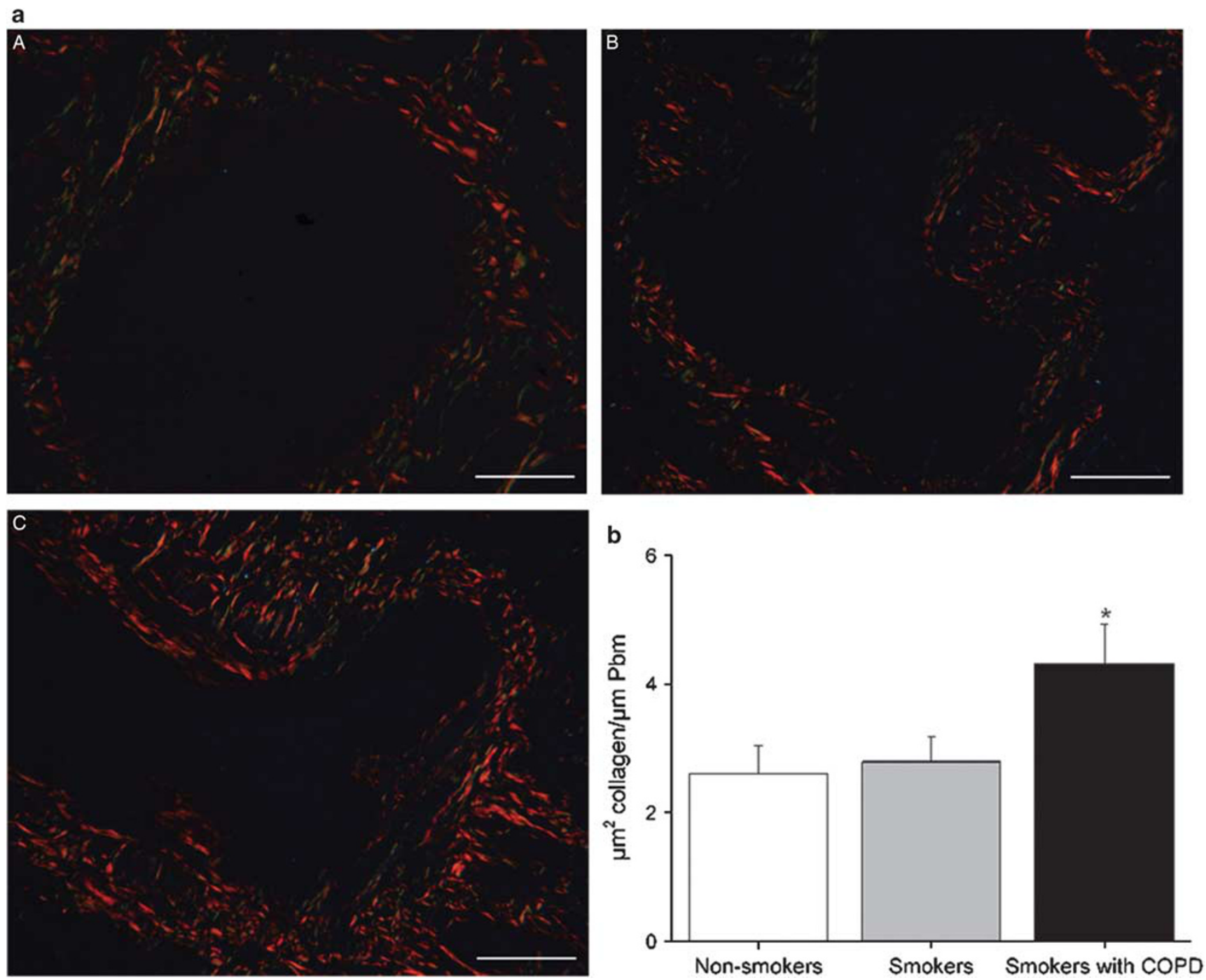

Figure 2 Collagen deposition in small airways from non-smokers, smokers and smokers with COPD. (a) Photomicrographs of sirius red staining showing collagen expression around the small airway walls from non-smokers (A), smokers (B), and smokers with COPD (C) (all images are $\times 400$ magnification, scale bars $=50 \mu \mathrm{m}$ ). (b) Measurement of collagen deposition in small airways. Results are calculated by the area of collagen deposition normalized to the length of basement membrane $(\mathrm{Pbm}) .{ }^{*} P<0.01$, compared with non-smokers.

manner (Figure 8a). Cell viability after stimulation with various concentrations of WRW4 was studied by MTT assay. As shown in Figure 8b, WRW4 at a concentration of $\leq 10 \mu \mathrm{M}$ did not affect the viability of HFL-1 cells. Combined, our results indicate that FPRL1 is required for LL-37-induced collagen production in HFL-1 cells.

\section{Increased ERK Phosphorylation in LL-37-Induced Collagen Production}

To assess whether mitogen-activated protein kinase (MAPK) pathways are involved in LL-37-induced collagen production, HFL-1 cells were pretreated with specific pathway inhibitors before synthetic LL-37 stimulation. We found that ERK inhibitor PD98059 effectively inhibited LL-37-induced collagen production. However, p38 inhibitor or JNK inhibitor did not exhibit any effect on LL-37-induced collagen expression (Figure 9a). These results suggest that ERK pathway is essential for LL-37-induced collagen production.

Furthermore, the effect of LL-37 on activation of ERK was evaluated by western blotting. As shown in Figure 9b, stimulation of HFL- 1 cells with synthetic LL-37 resulted in a transient phosphorylation of ERK with maximal phosphorylation observation at $30 \mathrm{~min}$. In addition, LL-37 activated ERK phosphorylaton in a dose-dependent manner (Figure 9c), which was sensitive to the specific inhibitor PD98059 (Figure 9d). We also observed that ERK phosphorylation was significantly inhibited by WRW4 (Figure 9d), suggesting that LL-37-induced ERK activation was FPRL1 dependent. Together, our data support the conclusion that LL-37 upregulates collagen expression in HFL-1 cells via activation of FPRL1-mediated ERK pathway. 


\section{a}
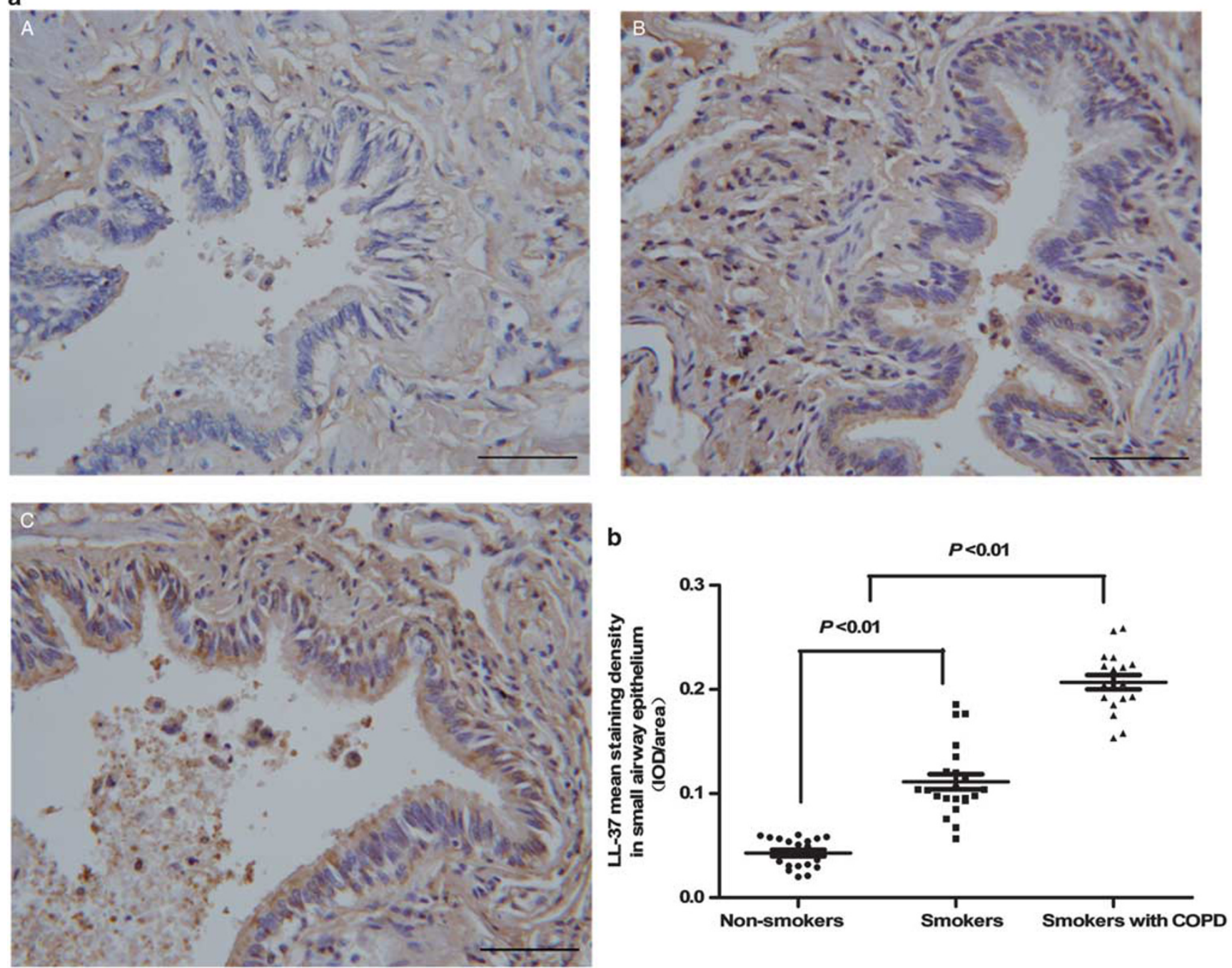

Figure 3 Expression of LL-37 in small airways from non-smokers, smokers, and smokers with COPD. (a) Photomicrographs of immunohistochemistry for LL-37 in small airways from non-smokers (A), smokers (B), and smokers with COPD (C) (all images are $\times 400$ magnification, scale bars $=50 \mu$ m). (b) Quantification of LL-37 immunoreactivity in small airway epithelium (mean staining density).
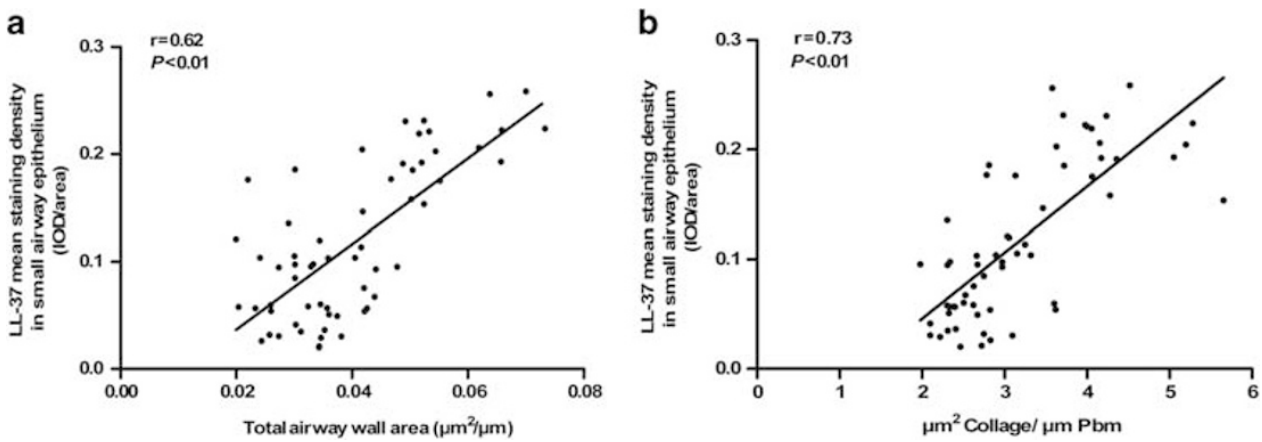

Figure 4 Correlation between the expression of LL-37 in small airway epithelium and structural changes of small airway remodeling. (a) LL-37 immunoreactivity in epithelium showed a significant correlation with the airway wall thickness. (b) LL-37 immunoreactivity in epithelium was positively correlated with the deposition of collagen in the airway wall. 

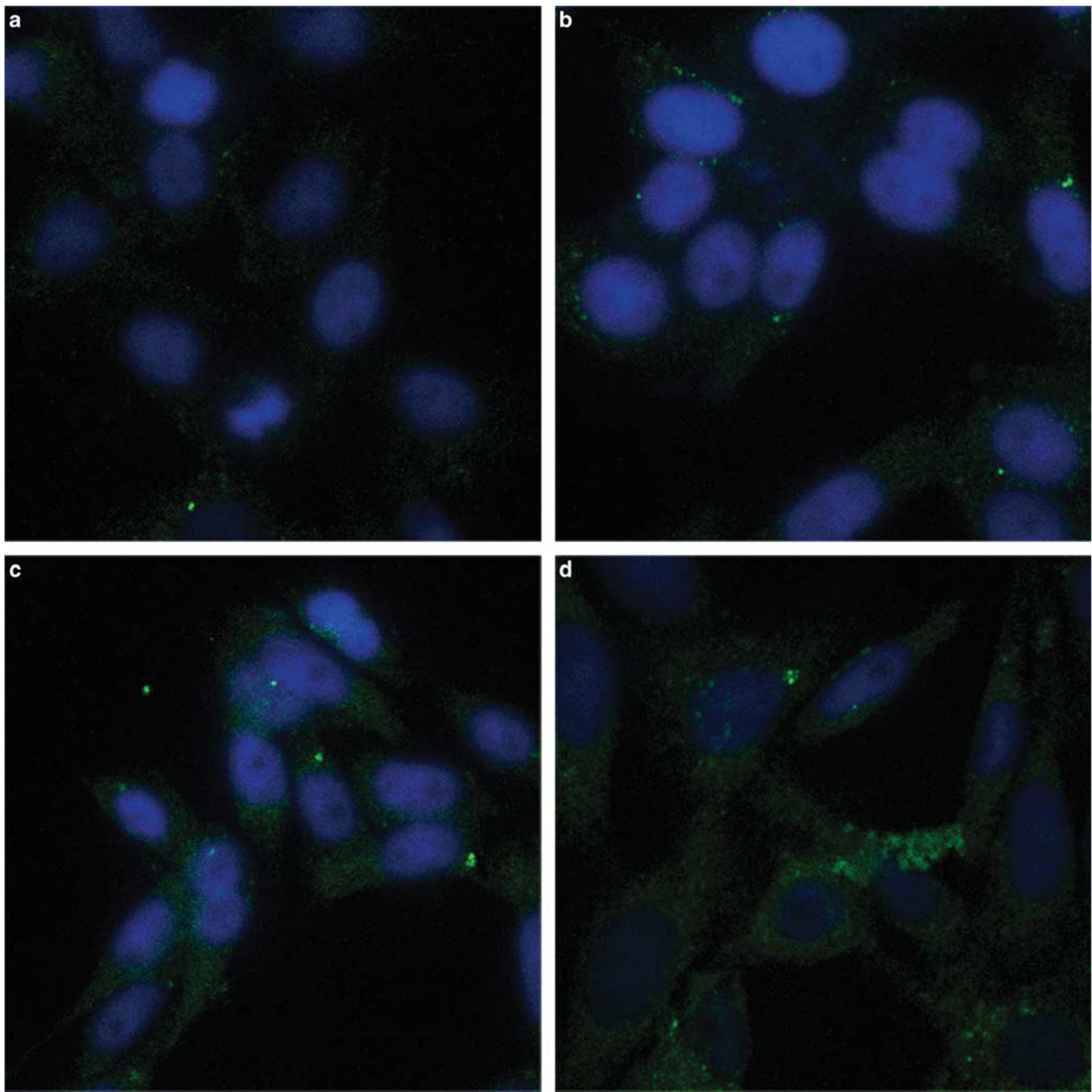

d

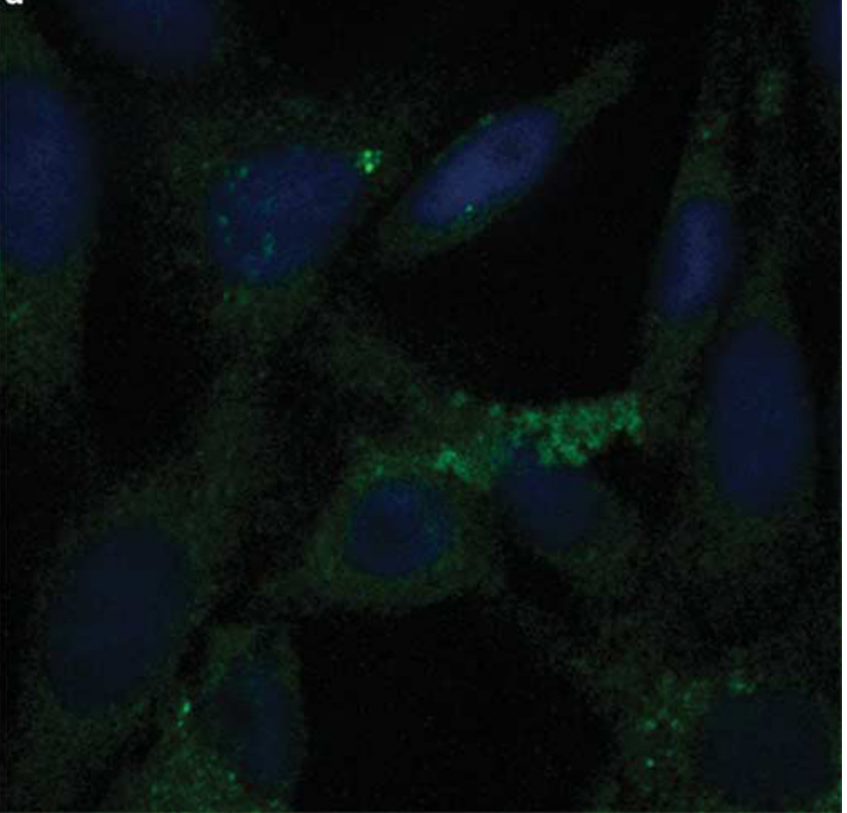

Figure 5 CSE increased LL-37 expression in 16HBE cells. 16HBE cells were treated with media alone (control) and various concentrations of CSE for $48 \mathrm{~h}$, and LL-37 expression was detected by immunofluorescence. (a) control, (b) CSE (2\%), (c) CSE (4\%), (d) CSE (6\%).

\section{DISCUSSION}

Results presented in this study showed that the immunoreactivity of LL-37 in small airway epithelium was significantly increased in smokers with COPD. Importantly, the expression of LL-37 in airway epithelium was correlated with airway wall thickness as well as deposition of collagen in the airway walls. In vitro, CSE elevated LL-37 expression in bronchial epithelial cells and epithelium-derived LL-37 augmented fibroblast collagen production. Additionally,
FPRL1-mediated ERK signaling pathway was involved in LL-37-induced collagen production in lung fibroblasts. Taken together, these results demonstrate that increased levels of LL-37 in airway epithelium after cigarette smoke exposure could stimulate collagen production in lung fibroblasts. This may add to the small airway remodelling in COPD.

Small airway remodeling is an important anatomical cause of airflow limitation in COPD. To date, however, the pathogenesis of small airway remodeling in COPD is an area 
a

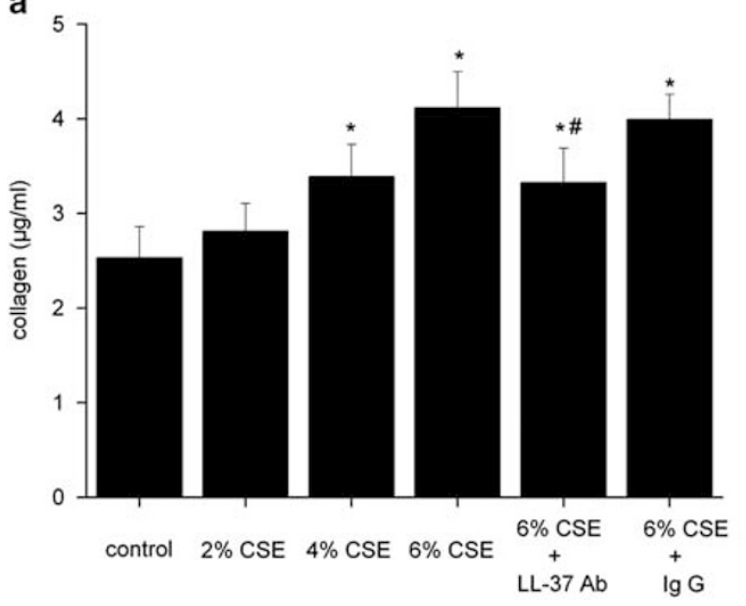

b

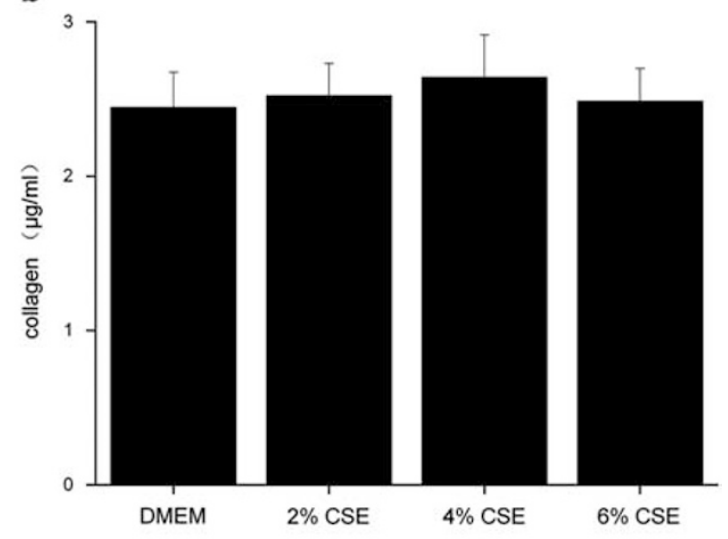

Figure 6 CSE-induced epithelial secretion of LL-37 upregulated fibroblast collagen production. (a) 16HBE and HFL-1 cells were co-cultured in six-well plates without direct contact. After exposure to CSE for $48 \mathrm{~h}$, total collagen production was elevated in a dose-dependent manner. LL-37 antibody significantly abolished collagen production, while anti-lgG had no effect on collagen production. ${ }^{*} P<0.01$, compared with the control group. ${ }^{\#} P<0.01$, compared with $6 \%$ CSE group. (b) CSE exposure for $48 \mathrm{~h}$ has no significant effect on collagen production in HFL-1 cells.
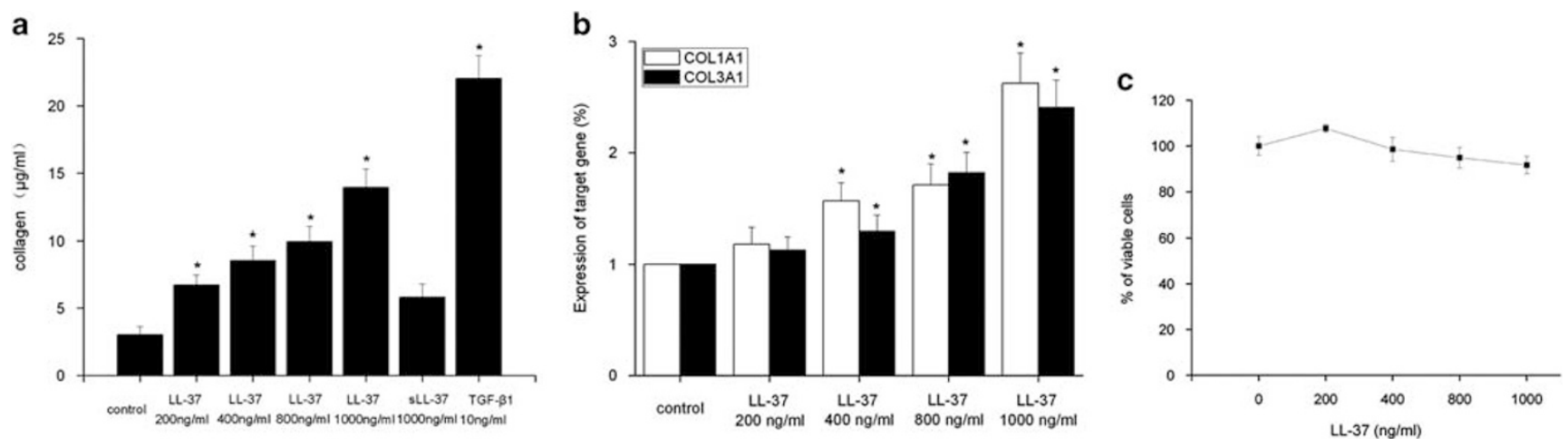

Figure 7 Synthetic LL-37 enhanced total collagen and collagen gene expression in human fetal lung fibroblasts (HFL-1). (a) Cells were treated with synthetic LL-37, transforming growth factor- $\beta 1$ (TGF- $\beta 1$ ), and scrambled LL-37 (sLL-37) for $48 \mathrm{~h}$. Total collagen in supernatants was detected by Sircol assay. ${ }^{*} P<0.01$ vs control group. (b) Cells were treated with synthetic LL-37 for $48 \mathrm{~h}$, total RNA was extracted, and RT-PCR was performed. Results are expressed as a ratio with glyceraldehydes-3-phosphate dehydrogenase (GAPDH) mRNA. ${ }^{*} P<0.01$ vs control group. (c) Cells were treated with synthetic LL-37 for $48 \mathrm{~h}$, after which cell viability was measured with MTT assay.

of debate. Some investigators have assumed that cigarette smoke-induced inflammatory response is responsible for small airway remodeling. ${ }^{5}$ However, this view has been challenged as some findings showed that attenuation of airway inflammation had no effect on airway remodeling, implying small airway remodeling was not a downstream effect of the pulmonary inflammation. ${ }^{30}$ Furthermore, increasing body of literature demonstrates that the remodeling processes of the small airways may attribute to excessive production of growth factors by airway epithelium. ${ }^{4,31}$

Based on the previous findings that LL-37 levels in small airway epithelium of COPD patients were significantly higher than control subjects, ${ }^{20}$ we began to investigate whether epithelial production of LL-37 was involved in small airway remodeling in COPD. In line with our previous findings, a marked upregulation of LL-37 expression was observed in small airways from COPD smokers compared with nonsmokers and smokers without COPD. As the most characteristic structural changes of small airway remodeling are thickening of the airway wall and increased collagen deposition, we compared the expression of LL-37 in epithelium with airway wall thickness and collagen deposition. Among all the subjects, the expression of LL-37 in epithelium correlated closely with airway wall thickness and deposition of collagen. These data suggest that epithelial expression of LL-37 may be associated with small airway remodeling, a finding that extends our previous work and could help elucidate the mechanism of small airway remodeling in COPD.

In our study, we observed that the levels of LL-37 in airway epithelium from smokers are higher than non-smokers, suggesting that cigarette smoke may account for the increased expression of LL-37. To clarify this point in vitro, we exposed 
a

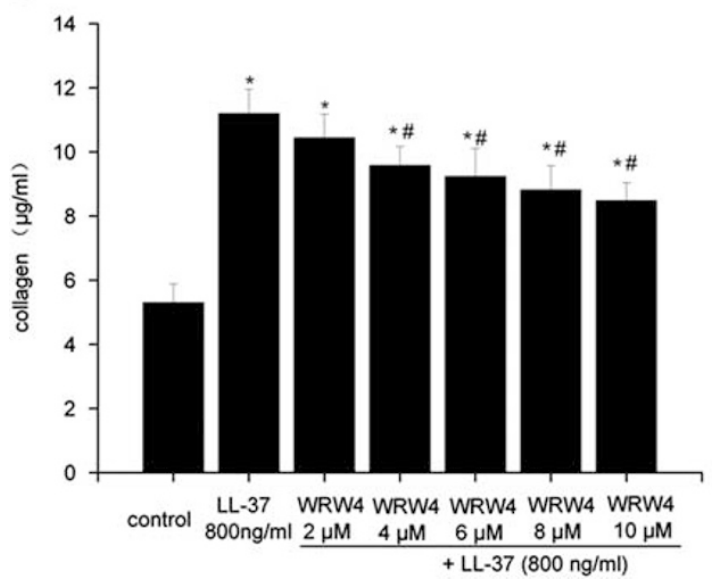

b

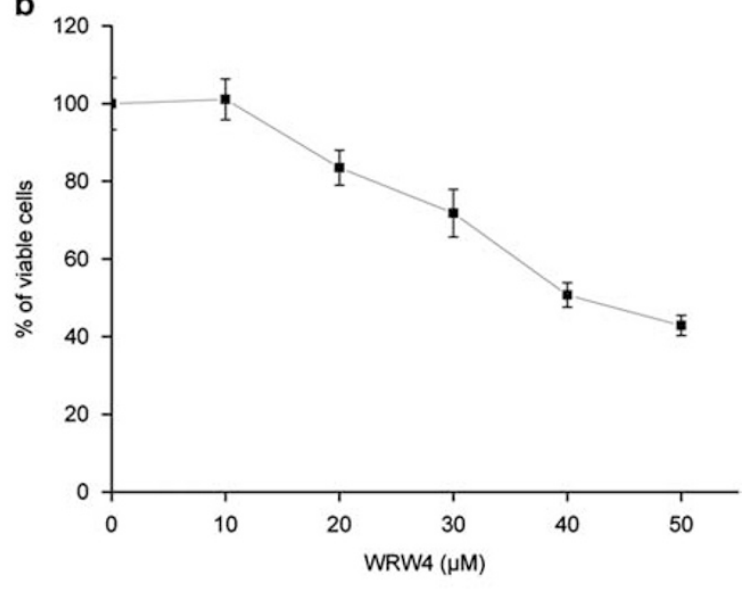

Figure 8 Formyl peptide receptor-like 1 (FPRL1) was involved in LL-37-induced collagen expression. (a) Human fetal lung fibroblasts (HFL-1) were preincubated with of FPRL1 antagonist WRW4 $2 \mathrm{~h}$ before addition of synthetic LL-37 $(800 \mathrm{ng} / \mathrm{ml})$. Total soluble collagen in supernatants was determined $48 \mathrm{~h}$ after stimulation. ${ }^{*} P<0.01$ compared with the control group. ${ }^{\#} P<0.05$ compared with the LL-37-treated group. (b) Cells were treated with WRW4 for $48 \mathrm{~h}$, after which cell viability was measured with MTT assay.

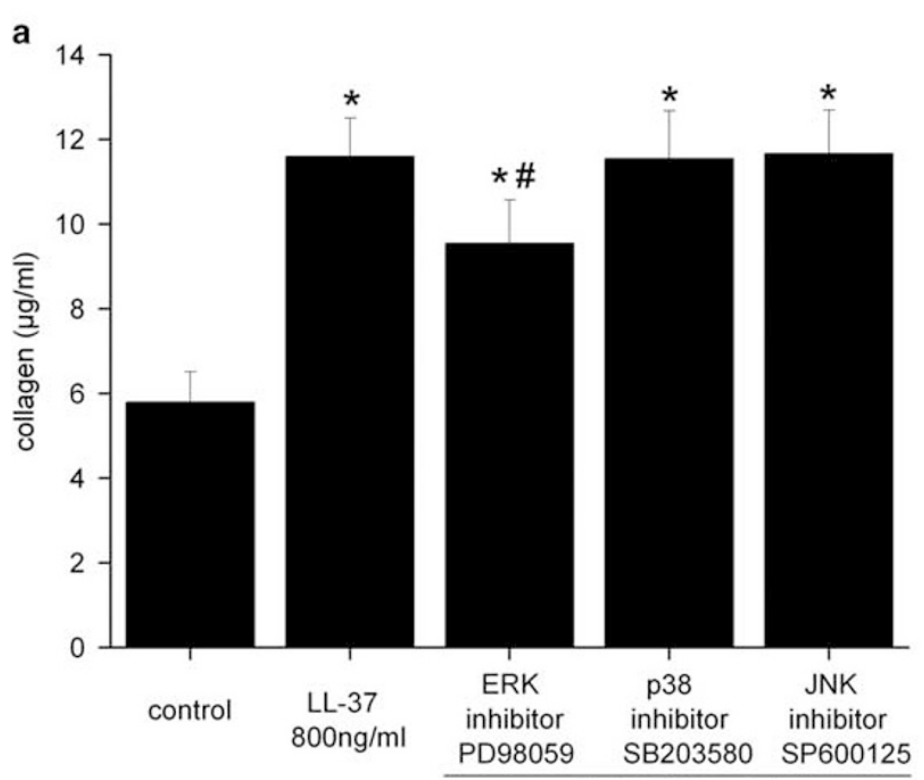

$+\mathrm{LL}-37(800 \mathrm{ng} / \mathrm{ml})$ b

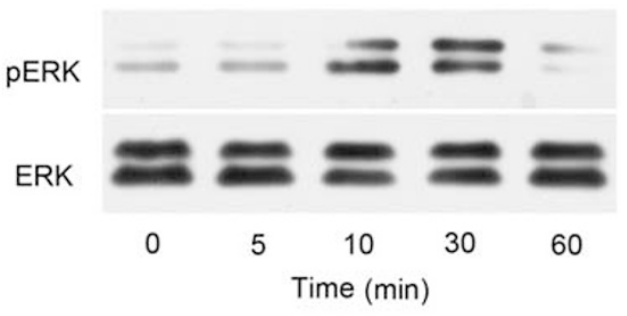

C

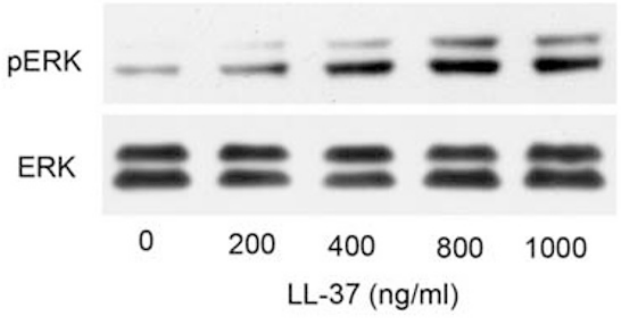

d

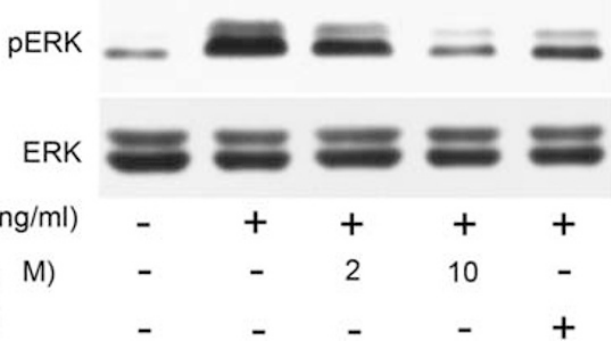

Figure 9 LL-37-induced collagen expression was dependent on extracellular signal-regulated kinase (ERK) activity. (a) Cells were treated with $10 \mu \mathrm{M}$ of extracellular signal-regulated kinase (ERK) inhibitor PD98059, $10 \mu \mathrm{M}$ of p38 inhibitor SB203580, or $20 \mu \mathrm{M}$ of JNK inhibitor SP600125 for $2 \mathrm{~h}$ and then stimulated with synthetic LL-37 $(800 \mathrm{ng} / \mathrm{ml})$. After $48 \mathrm{~h}$, total soluble collagen was detected by Sircol assay, each performed in duplicate. Data are expressed as mean \pm s.d. ${ }^{*} P<0.01$ vs control group, ${ }^{\#} P<0.01$ vs LL-37 treated group. (b) Cells were treated with $800 \mathrm{ng} / \mathrm{ml}$ of synthetic LL-37 for the indicated time periods. Phosphorylation of ERK (pERK) and total ERK were analyzed by western blotting. (c) Cells were treated with the indicated concentrations of synthetic LL-37 for $30 \mathrm{~min}$. Cell extracts were subjected to western blotting analysis to detect pERK and total ERK. (d) Cells were pretreated with WRW4 $(2 \mu \mathrm{M}$ or $10 \mu \mathrm{M})$ or PD98059 $(10 \mu \mathrm{M}) 2 \mathrm{~h}$ before stimulation of synthetic LL-37 $(800 \mathrm{ng} / \mathrm{ml})$. After $30 \mathrm{~min}$, pERK and total ERK were analyzed by western blotting. 
16HBE cells with different concentrations of CSE and found that CSE leads to a significant elevation of LL-37 in 16HBE cells, which is in accordance with our previous study that CSE enhanced LL-37 expression in human bronchial epithelial cells and alveolar epithelial cells. ${ }^{20}$ Similarly, $\alpha$-defensin was also reported to be induced by CSE in human alveolar epithelial cells and gingival epithelial cells, ${ }^{32,33}$ indicating a potential effect of cigarette smoke on AMP's expression in epithelial cells.

Airway epithelium, beyond its role as a physical barrier, can also interact with underlying mesenchymal cells functionally, which is referred to as the epithelial-mesenchymal trophic unit (EMTU).$^{34}$ It has been demonstrated that TGF- $\beta$ is released from damaged epithelial cells and induces the transformation of fibroblasts via TGF- $\beta$ receptors. ${ }^{34}$ A study in transgenic mice also suggested that expression of a TGF- $\alpha$ gene in the respiratory epithelial cells led to lung fibrosis. ${ }^{35}$ Nevertheless, it remains unknown whether epithelial production of LL-37 can influence the function of subepithelial lung fibroblasts. In this study, we established a co-culture model to mimic EMTU in vitro. Though direct cell-to-cell contacts were prevented by a $0.4-\mu \mathrm{m}$ plastic membrane, soluble substances could communicate between the upper and the lower cells. We observed that CSE did not influence collagen production significantly; however, CSE-induced epithelial secretion enhanced fibroblast collagen production. Additionally, LL-37 was among the factors involved in this effect, based on the observation that LL-37 neutralizing antibody significantly decreased collagen production. These results demonstrate that LL-37 is an important regulator of EMTU by means of enhancing fibroblast collagen production, providing in vitro evidence for an association between LL-37 overproduction in airway epithelium and collagen deposition in small airways.

On the basis of the above findings, we were prompted to further explore the role of LL-37 in collagen production in lung fibroblasts in vitro. As expected, synthetic LL-37 elevated soluble collagen production and collagen gene expression in HFL-1 cells in a dose-dependent manner, confirming the capacity of LL-37 to stimulate collagen synthesis in lung fibroblasts. Our results were consistent with previous work demonstrating that AMPs are able to increase collagen production in fibroblasts. For instance, human neutrophil peptide-1 was demonstrated to enhance the expression of type I collagen in human dermal fibroblasts. ${ }^{36}$ Yoshioka $e t ~ a l^{37}$ also showed that $\alpha$-defensin induced collagen expression in human lung fibroblasts.

LL-37 is known to have various biological effects mediated via FPRL1, such as immunomodulatory and chemotactic activities. $^{38,39}$ FPRL1 is a seven-transmembrane, G proteincoupled receptor. Although predominately expressed in phagocytic leukocytes, functional FPRL1 is also expressed on human lung and skin fibroblasts. ${ }^{40}$ In this study, we demonstrated that FPRL1 participated in LL-37-induced collagen production in human lung fibroblasts, as shown by the ability of the FPRL1 antagonist WRW4, at noncytotoxic concentrations, to attenuate LL-37-induced collagen production in a dose-dependent manner. In this regard, FPRL1 on lung fibroblasts may be an important binding site for epithelial-derived LL-37. Moreover, FPRL1 antagonist might be a possible therapeutic target in the treatment and prevention of excessive collagen deposition in small airway remodeling of COPD.

Given the involvement of FPRL1 in LL-37-induced collagen production, we sought to determine the key signaling mechanisms by which this occurs. It is well established that FPRL1 stimulation leads to activation of MAPK signal transduction pathways, ${ }^{41}$ which have important roles in regulating collagen production in cardiac fibroblasts and human mesangial cells. ${ }^{42,43}$ In this study, we demonstrate that ERK signaling is required for LL-37-induced collagen production in HFL-1 cells. This proposal is suggested by the effect that ERK blockade by PD98059 significantly inhibits LL-37-induced collagen expression. Additionally, LL-37 activated ERK phosphorylation, and this effect was inhibited by WRW4, suggesting that ERK is the downstream pathway of FPRL1. Taken as a whole, we presume that LL-37, a product of airway epithelial cells in response to cigarette smoke exposure, could bind to FPRL1 on fibroblasts, activate its downstream ERK pathway, and promote collagen production, which may enhance the progression of small airway remodeling in COPD.

In summary, a close association was found between the expression of LL-37 in airway epithelium and the structural changes of small airway remodeling in COPD. In vitro, we showed that CSE-induced epithelial secretion of LL-37 increased fibroblast collagen production. Furthermore, FPRL1dependent ERK pathway was involved in LL-37-induced fibroblast collagen production. These findings demonstrate that LL-37 in airway epithelium may play an important role in the pathological changes of small airway remodeling in COPD.

\section{ACKNOWLEDGMENTS}

This study was supported by Grants from the National Science Foundation of China (No. 81170041).

\section{DISCLOSURE/CONFLICT OF INTEREST}

The authors declare no conflict of interest.

1. Pauwels R. Global initiative for chronic obstructive lung diseases (GOLD): time to act. Eur Respir J 2001;18:901-902.

2. Hogg JC, Macklem PT, Thurlbeck WM. Site and nature of airway obstruction in chronic obstructive lung disease. N Engl J Med 1968; 278:1355-1360.

3. Shaw RJ, Djukanovic R, Tashkin DP, et al. The role of small airways in lung disease. Respir Med 2002;96:67-80.

4. Takizawa $\mathrm{H}$, Tanaka M, Takami $\mathrm{K}$, et al. Increased expression of transforming growth factor- $\beta 1$ in small airway epithelium from tobacco smokers and patients with chronic obstructive pulmonary disease. Am J Respir Crit Care Med 2001;163:1476-1483.

5. Jeffery PK. Remodeling in asthma and chronic obstructive lung disease. Am J Respir Crit Care Med 2001;164:S28-S38. 
6. Chapman HA. Disorders of lung matrix remodeling. J Clin Invest 2004;113:148-157.

7. Golec M. Cathelicidin LL-37: LPS-neutralizing, pleiotropic peptide. Ann Agric Environ Med 2007;14:1-4.

8. Matsushita I, Hasegawa K, Nakata K, et al. Genetic variants of human $\beta$ defensin-1 and chronic obstructive pulmonary disease. Biochem Biophys Res Commun 2002;291:17-22.

9. Doss $M$, White MR, Tecle $T$, et al. Human defensins and LL-37 in mucosal immunity. J Leukoc Biol 2010;87:79-92.

10. Sørensen O, Arnljots K, Cowland JB, et al. The human antibacterial cathelicidin, hCAP-18, is synthesized in myelocytes and metamyelocytes and localized to specific granules in neutrophils. Blood 1997;90:2796-2803.

11. Bals R, Wang X, Zasloff M, et al. The peptide antibiotic LL-37/hCAP-18 is expressed in epithelia of the human lung where it has broad antimicrobial activity at the airway surface. Proc Natl Acad Sci USA 1998;95:9541-9546.

12. Frohm $M$, Agerberth $B$, Ahangari $G$, et al. The expression of the gene coding for the antibacterial peptide LL-37 is induced in human keratinocytes during inflammatory disorders. J Biol Chem 1997;272:15258-15263.

13. Hase K, Eckmann L, Leopard JD, et al. Cell differentiation is a key determinant of cathelicidin LL-37/human cationic antimicrobial protein 18 expression by human colon epithelium. Infect Immun 2002; 70:953-1063.

14. Ciornei $C D$, Sigurdardóttir T, Schmidtchen A, et al. Antimicrobial and chemoattractant activity, lipopolysaccharide neutralization, cytotoxicity, and inhibition by serum of analogs of human cathelicidin LL-37. Antimicrob Agents Chemother 2005;49:2845-2850.

15. Koczulla $R$, von Degenfeld $G$, Kupatt $C$, et al. An angiogenic role for the human peptide antibiotic LL-37/hCAP-18. J Clin Invest 2003;111:1665-1672.

16. Shaykhiev R, Beißwenger $C$, Kändler $K$, et al. Human endogenous antibiotic LL-37 stimulates airway epithelial cell proliferation and wound closure. Am J Physiol Lung Cell Mol Physiol 2005;289:L842-L848.

17. Golec M, Reichel C, Mackiewicz B, et al. Cathelicidin LL-37, granzymes, TGF- $\beta 1$ and cytokines levels in induced sputum from farmers with and without COPD. Ann Agric Environ Med 2009;16:289-297.

18. Xiao W, Hsu YP, Ishizaka A, et al. Sputum cathelicidin, urokinase plasminogen activation system components, and cytokines discriminate cystic fibrosis, COPD, and asthma inflammation. Chest 2005;128: 2316-2326.

19. Golec M, Reichel C, Lemieszek M, et al. Cathelicidin LL-37 in bronchoalveolar lavage and epithelial lining fluids from COPD patients and healthy individuals. J Biol Regul Homeost Agents 2012;26:617-625.

20. Jiang YY, Xiao W, Zhu MX, et al. The effect of human antibacterial peptide LL-37 in the pathogenesis of chronic obstructive pulmonary disease. Respir Med 2012;106:1680-1689.

21. Zhang YK, Jiang YY, Sun CC, et al. The human cathelicidin LL-37 enhances airway mucus production in chronic obstructive pulmonary disease. Biochem Biophys Res Commun 2014;443:103-109.

22. Park HJ, Cho DH, Kim HJ, et al. Collagen synthesis is suppressed in dermal fibroblasts by the human antimicrobial peptide LL-37. J Invest Dermatol 2009;129:843-850.

23. Vestbo J, Hurd SS, Agustí AG, et al. Global strategy for the diagnosis, management, and prevention of chronic obstructive pulmonary disease: GOLD executive summary. Am J Respir Crit Care Med 2013;187:347-365.

24. Cao $Y$, Zeng $D$, Song $Q$, et al. The effects of antisense interleukin- 4 gene transferred by recombinant adeno-associated virus vector on the airway remodeling in allergic rats. J Asthma 2010;47:951-958.
25. Jia XL, Li SY, Danq SS, et al. Increased expression of chondroitin sulphate proteoglycans in rat hepatocellular carcinoma tissues. World J Gastroenterol 2012;18:3962-3976.

26. Tsui L, Fong TH, Wang IJ. YC-1 targeting of hypoxia-inducible factor- $1 \alpha$ reduces RGC-5 cell viability and inhibits cell proliferation. Mol Vis 2012;18:1594-1603.

27. Noguchi C, Umino T, Miyazaki Y, et al. TGF- $\beta$ and glutathione promote tissue repair in cigarette smoke induced injury. J Med Dent Sci 2007;54:109-116.

28. Aoudjehane L, Pissaia A, Scatton $\mathrm{O}$, et al. Interleukin-4 induces the activation and collagen production of cultured human intrahepatic fibroblasts via the STAT-6 pathway. Lab Invest 2008;88:973-985.

29. Kim TY, Lee HJ, Hwang KS, et al. Methylation of RUNX3 in various types of human cancers and premalignant stages of gastric carcinoma. Lab Invest 2004;84:479-484.

30. Bracke KR, D'hulst Al, Maes $\mathrm{T}$, et al. Cigarette smoke-induced pulmonary inflammation, but not airway remodelling, is attenuated in chemokine receptor 5-deficient mice. Clin Exp Allergy 2007;37: 1467-1479.

31. Aubert JD, Hayashi S, Hards J, et al. Platelet-derived growth factor and its receptor in lungs from patients with asthma and chronic airflow obstruction. Am J Physiol 1994;266:L655-L663.

32. Pierson $T$, Learmonth-Pierson $S$, Pinto $D$, et al. Cigarette smoke extract inducesdifferential expression levels of $\beta$-defensin peptides in human alveolar epithelial cells. Tob Induc Dis 2013;11:10.

33. Semlali A, Witoled C, Alanazi $M$, et al. Whole cigarette smoke increased the expression of TLRs, HBDs, and proinflammory cytokines by human gingival epithelial cells through different signaling pathways. PLoS One 2012;7:e52614.

34. Boxall C, Holgate ST, Davies DE. The contribution of transforming growth factor- $\beta$ and epidermal growth factor signalling to airway remodelling in chronic asthma. Eur Respir J 2006;27:208-229.

35. Korfhagen TR, Swantz RJ, Wert SE, et al. Respiratory epithelial cell expression of human transforming growth factor- $\alpha$ induces lung fibrosis in transgenic mice. J Clin Invest 1994;93:1691-1699.

36. Oono T, Shirafuji Y, Huh WK, et al. Effects of human neutrophil peptide- 1 on the expression of interstitial collagenase and type I collagen in human dermal fibroblasts. Arch Dermatol Res 2002;294: 185-189.

37. Yoshioka $\mathrm{S}$, Mukae $\mathrm{H}$, Ishii $\mathrm{H}$, et al. $\alpha$-Defensin enhances expression of HSP47 and collagen-1 in human lung fibroblasts. Life Sci 2007; 80:1839-1845.

38. Scott MG, Davidson DJ, Gold MR, et al. The human antimicrobial peptide LL-37 is a multifunctional modulator of innate immune responses. J Immunol 2002;169:3883-3891.

39. Yang D, Chen $\mathrm{Q}$, Schmidt AP, et al. LL-37, the neutrophil granule-and epithelial cell-derived cathelicidin, utilizes formyl peptide receptor-like 1 (FPRL1) as a receptor to chemoattract human peripheral blood neutrophils, monocytes, and T cells. J Exp Med 2000;192:1069-1074.

40. VanCompernolle $\mathrm{SE}$, Clark KL, Rummel $\mathrm{KA}$, et al. Expression and function of formyl peptide receptors on human fibroblast cells. J Immunol 2003;171:2050-2056.

41. He R, Shepard LW, Chen J, et al. Serum amyloid A is an endogenous ligand that differentially induces IL-12 and IL-23. J Immunol 2006; 177:4072-4079.

42. Sato $M$, Shegogue $D$, Gore EA, et al. Role of $p 38$ MAPK in transforming growth factor $\beta$ stimulation of collagen production by scleroderma and healthy dermal fibroblasts. J Invest Dermatol 2002;118:704-711.

43. Kajanne R, Miettinen $P$, Mehlem A, et al. EGF-R regulates MMP function in fibroblasts through MAPK and AP-1 pathways. J Cell Physiol 2007:212:489-497. 